\title{
Quantification of Low Voltage Images of 2-dimensional Materials in Aberration Corrected Scanning Transmission Electron Microscopy.
}

\author{
Mark P. Oxley ${ }^{1}$, Nicholas G. Cross ${ }^{2}$, Gerd Duscher ${ }^{2}$, Leslie J. Allen ${ }^{3}$ and Matthew F. Chisholm ${ }^{1}$ \\ 1. Oak Ridge National Laboratory, Materials Science and Technology Division, Oak Ridge TN, USA. \\ 2. University of Tennessee, Materials Science and Engineering, Knoxville TN, USA. \\ 3. University of Melbourne, School of Physics, Parkville Victoria, Australia.
}

Quantitative analysis of high voltage scanning transmission electron microscope (STEM) images using annular dark field (ADF) has become routine [1]. Advances in aberration correction has extended atomic resolution imaging to lower incident electron energies suitable for the imaging of $2 \mathrm{D}$ materials such as $\mathrm{BN}$ and graphene [2,3]. Lower energies, such as $60 \mathrm{keV}$, reduce knock-on damage but in turn greatly increase the effects of temporal incoherence, leading to extended probe tails and reduced contrast. In addition, the signal to noise ratio of images of $2 \mathrm{D}$ materials is also quite low, since the electron beam is scattered by only a few atoms, rather than an extended atomic column. Even with lower energies, some specimens are still prone to beam damage and a series of fast scanned images is often averaged to obtain a useful image. This raises the issue of image alignment or registration. In this work we demonstrate strategies for correcting temporal incoherence, allowing true quantitative comparison of experiment and simulation.

In addition to temporal incoherence, residual aberrations also lead to a reduction in image contrast. In Figure 1 (a) we compare a perfect probe profile for $60 \mathrm{keV}$ incident electrons formed with a 30 mrad. probe forming aperture (black line) with the profile of a probe containing residual aberrations (red line). This results in a peak intensity reduction of approximately one third. Because residual aberrations are dominated by non-circular aberrations, the probe profile is also no longer symmetric. The addition temporal incoherence results in a further reduction in peak intensity to around $50 \%$ of the original value. This intensity has been pushed out into the probe tails. In Figure 1 (b) and (c) we compare simulated ADF images of BN for a perfect probe (b) and an aberrated incoherent probe (c). Both images are shown on the same overall scale. The reduction of contrast is obvious. Clearly a simple "source size" correction, as used in earlier high voltage microscopy quantification, will not allow true quantification of these images [1].

In order to minimize specimen beam damage, it is common to acquire a time series of fast scan images and average over these images in order to increase the signal to noise ratio (SNR). Even with the low specimen drifts achieved in the Nion UltraSTEM, careful image alignment is the first step towards true quantification. In Figure 2 (a) we show a summed stack of $20 \mathrm{ADF}$ images of $\mathrm{MoS}_{2}$ with W and Se impurities taken on the UltraSTEM operating at $60 \mathrm{keV}$. There is clearly minimal drift since atomic locations are clearly seen. In order to improve the image, we have applied first rigid registration followed by a non-rigid demons algorithm. Initial results are shown in Figure 2 (b). We have used the registration algorithms available in the SimpleITK package [4]. While the image has improved SNR we still need to correct for temporal incoherence and residual aberrations.

Temporal incoherence results in a defocus spread in the incident probe and can only be included exactly by summing over a properly weighted distribution of images calculated for these different defocus values. We aim to correct image intensities to allow quantitative comparison with simulations using readily 
available software packages. We may write the distorted probe as $\tilde{P}(\mathbf{R})=F(\mathbf{R}) \otimes P(\mathbf{R})$ where $P$ is the perfect probe and $F$ a function describing the aberrations and incoherence. It can be found simply by deconvolving the perfect probe from the aberrated/incoherent probe. Using a phase object approximation (reasonable for a 2D material), and some other reasonable approximations, it can be shown that the aberrated/incoherent image can be written $\tilde{I}(\mathbf{R})=F(-\mathbf{R}) \otimes I(\mathbf{R})$ where $I$ is the image that would be formed with a perfect probe. This image can then be found by deconvolving $F(-\mathbf{R})$ from the experimental image. The result of such a deconvolution is shown in Figure 2 (c) leading to higher contrast and improved image localization. We will combine this method with image simulation in order to unambiguously identify impurities and defect in 2D materials.

\section{References:}

[1] J. M. LeBeau et al, Physical Review Letters, 100, (2008) 206101.

[2] O. L. Krivanek et al., Nature 462 (2010), p. 1342.

[3] W. Zhou et al., Microscopy and Microanalysis, 18 (2012), p. 342.

[4] B. C. Lowekamp et al., Frontiers in Neuroinformatics, 7 (2013), p. 45.

[5] This research was supported by the US Department of Energy, Office of Basic Energy Sciences, Materials Sciences and Engineering Division. N.C. and G.D. acknowledge support from the National Science Foundation (NSF) under Grant No. DMR-1410940.
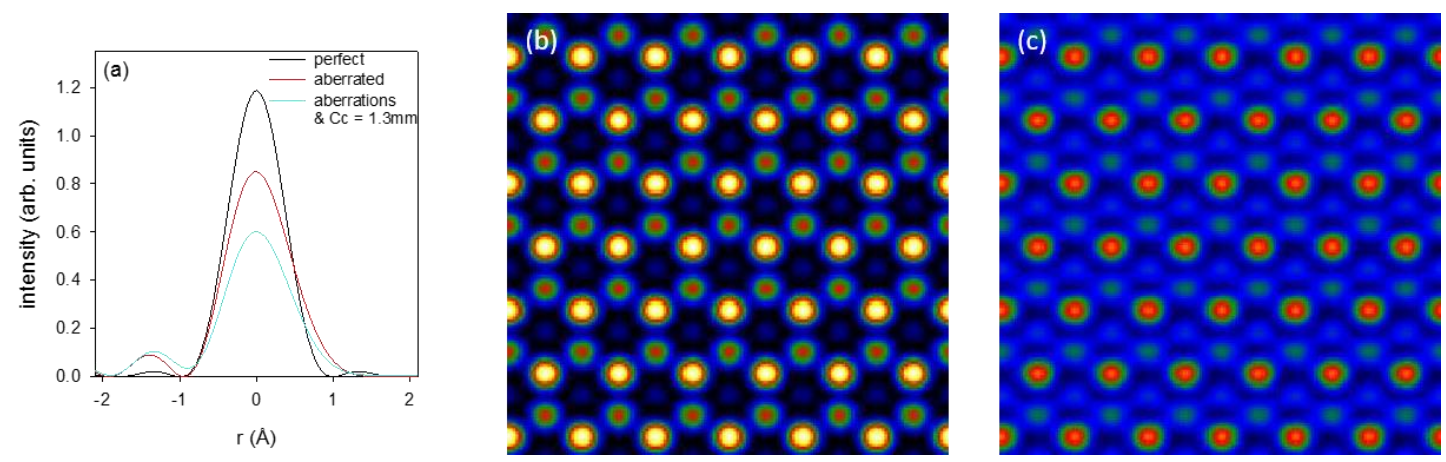

Figure 1. (a) $60 \mathrm{keV}$ probes formed with a $30 \mathrm{mrad}$ probe forming aperture for a perfect probe (black line), a probe formed with residual aberrations (red line) and including aberrations and and incoherence (cyan line). (b) Simulated ADF images of BN using a perfect probe. (c) Simulated ADF images of BN using an aberrated probe including temporal incoherence.
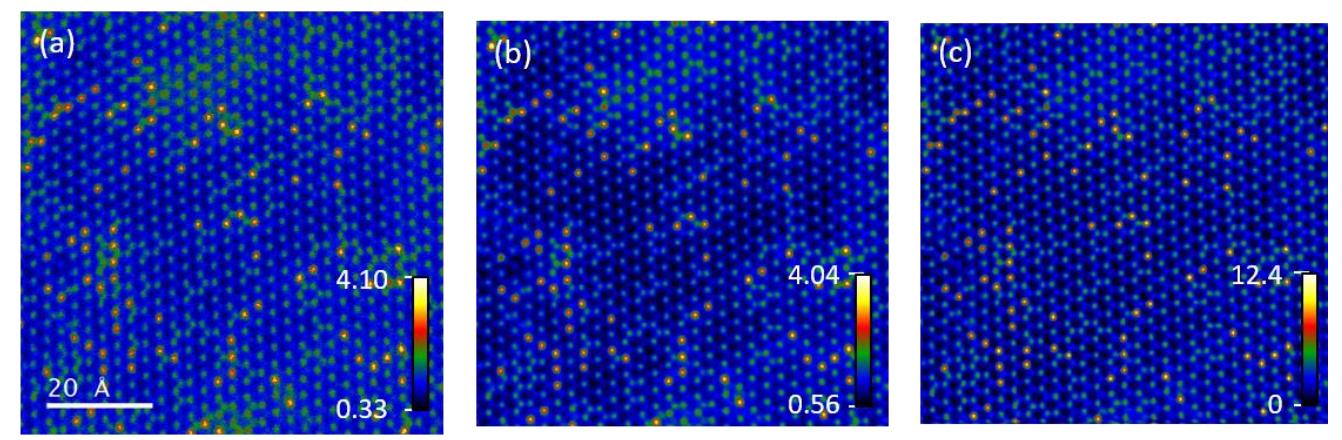

Figure 2. (a) Summed raw image stack of $\mathrm{MoS}_{2}$ with W and Se impurities. (b) Summed stack after nonrigid registration. (c) Image (b) corrected for temporal incoherence and residual aberrations. Images acquired at $60 \mathrm{kV}$ on ORNL's Nion UltraSTEM. 